\title{
Attribute Reduction in Property Oriented Concept Lattices of Formal Contexts and Formal Decision Contexts
}

\author{
Junyu Li, Zeng Qiang and Xia Wang ${ }^{1}$ \\ School of Mathematics, Physics and Information Science, Zhejiang Ocean \\ University \\ Key Laboratory of Oceanographic Big Data Mining and Application of Zhejiang \\ Province \\ Zhoushan, Zhejiang 316022, China \\ bblylm@126.com
}

\begin{abstract}
This paper studies notions and approaches to attribute reduction in property oriented concept lattices of formal contexts and formal decision contexts based on congruence relations. Firstly, dependence space based on the property oriented concept lattice is researched to obtain the relationship among property oriented concept lattices and the corresponding congruence relations. Then notions of attribute reduction is defined for formal contexts and decision contexts to find minimal attribute subsets which can preserve all congruence classes determined by the original attribute set, and also keep all property oriented extents and their original hierarchy in the property oriented concept lattice. Finally, approaches of discernibility matrix are presented to calculate all attribute reducts.
\end{abstract}

Keywords: Property oriented concept lattice, attribute reduction, congruence relation, formal context, formal decision context

\section{Introduction}

Knowledge reduction for formal concept analysis [1-2] can make knowledge representation of database more succinct, knowledge hiding in database clearer, and adaptability of rule sets for decision tables better. Many types of approaches to knowledge reduction for formal concept analysis have been proposed [1,3-7,9-17]. Some of these approaches consider knowledge reduction from the viewpoint of the extents of formal concepts and their hierarchy. For example, an attribute reduction approach was presented to find minimal attribute sets which can determine all extents and their original hierarchy in the concept lattice in [17-18]. And attribute reduction in a consistent formal decision context was also investigated in [15]. Wang et al. [9-10] provided another approach to attribute reduction, which only required to preserve all extents of $\wedge$-irreducible elements, and this method also preserves all extents and their original hierarchy in the concept lattice all extents and their original hierarchy in the concept lattice. Other approaches have been also developed for knowledge reduction for formal concept analysis on the basis of different criteria. For example, the reducible attribute and reducible object were proposed from the viewpoint of shortening lines or rows in [1]. Methods for attribute reduction were studied by an order-preserving mapping between the set of all the extents of the condition concept lattice and that of the decision concept lattice in [3]. In [4], methods of approximate concept construction were presented for an incomplete formal context. In [5] an efficient post-processing method was shown to prune redundant rules by virtue of the property of Galois connection, which inherently

${ }^{1}$ Corresponding author: Xia Wang; E-mail: bblylm@126.com1 
constrains rules with respect to objects. In [7] a Boolean approach was formulated to calculate all reducts of a formal context via the use of discernibility function. Wu et al. [16] studied attribute reduction in formal contexts from the viewpoint of keeping granular structure of concept lattices. Wang et al. [11] developed an approach to attribute reduction in a formal context and a consistent formal decision context based on congruence relations. The approach in a formal context is to find minimal attribute sets which can preserve all original congruence classes. Four types of approaches to attribute reduction in inconsistent formal decision contexts were defined in [12]. In [13], a unified model is constructed for formal contexts, consistent formal decision contexts and inconsistent formal decision contexts, which is called a consistent approximate representation space.

Yao [19-20] introduced the notion of formal concept and formal concept lattice into rough set, and defined the object and property oriented formal concept lattices based on approximation operators. There are also some approaches of knowledge reduction in the object and property oriented formal concept lattices. In [6] the approach developed in [910] was generalized to attribute reduction in the attribute oriented concept lattices and the object oriented concept lattices. The approach used in [11] was extended to attribute reduction in object oriented concept lattices and property oriented concept lattices. Subsequently an approach to attribute reduction in an object oriented concept lattice of a formal context based on congruence relations in [14].

The purpose of this paper is to introduce the approach proposed in [11-12] into knowledge reduction in the property oriented concept lattice based on formal contexts and formal decision contexts respectively. Basic definitions and properties of formal concept analysis are recalled in Section 2. In Section 3, dependence space is introduced into the property oriented concept lattice to obtain relationships among property oriented concept lattices and the corresponding congruence relations. Then an approach to attribute reduction in formal contexts is also proposed using discernibility matrices in Section 3. In Section 4, notions and approaches to attribute reduction in formal decision contexts is discussed. Finally, we conclude the paper in Section 5.

\section{Preliminaries}

In this section, we recall some basic notions and properties about formal concept analysis which will be used in this paper.

Definition 1 ([1]) A formal context $(U, A, I)$ consists of object set $U$ and attribute set $A$, and a relation $I \subseteq U \times A$. The elements of $U$ are called objects and the elements of $A$ are called attributes of the formal context.

For any $X \subseteq U$ and $B \subseteq A$, Y.Y. Yao [20] defined two pairs of dual operators:

$X^{\square}=\{a \in A \mid \forall x \in U, x I a \Rightarrow x \in X\}, X^{\diamond}=\{a \in A \mid \exists x \in U, x I a \wedge x \in X\} ;$

$B^{\square}=\{x \in U \mid \forall a \in A, x I a \Rightarrow a \in B\}, \quad B^{\diamond}=\{x \in U \mid \exists a \in A, x I a \wedge a \in B\}$.

Definition 2 ([20]) Let $(U, A, I)$ be a formal context and $B \subseteq A$. The formal context $\left(U, B, I_{B}\right)$ is called a subcontext of $(U, A, I)$, where $I_{B}=I \cap(U \times B)$.

For any $B \subseteq A$, let ${ }^{\square B},{ }^{\diamond B}$ stand for the operator in the subcontext $\left(U, B, I_{B}\right)$. Clearly, for any $X \subseteq U, X^{\square A}=X^{\square}, X^{\square B}=X^{\square A} \cap B, X^{\diamond A}=X^{\diamond}$ and $X^{\diamond B}=X^{\diamond A} \cap B$.

Definition 3 ([20]) A property oriented concept of a formal context $(U, A, I)$ is a pair $(X, B){ }_{\text {with }} X \subseteq U, B \subseteq A, X=B^{\square}$ and $B=X^{\diamond}$. We call $X$ the extent and $B$ the intent of the property oriented concept $(X, B)$. 
For any two property oriented concepts $\left(X_{1}, B_{1}\right)$ and $\left(X_{2}, B_{2}\right)$, Y.Y. Yao defined two operators meet and join as follows:

$$
\begin{gathered}
\left(X_{1}, B_{1}\right) \wedge\left(X_{2}, B_{2}\right)=\left(X_{1} \cap X_{2},\left(X_{1} \cap X_{2}\right)^{\oslash}\right), \\
\left(X_{1}, B_{1}\right) \vee\left(X_{2}, B_{2}\right)=\left(\left(B_{1} \cup B_{2}\right)^{\square}, B_{1} \cup B_{2}\right) .
\end{gathered}
$$

The set of all property oriented concepts of $(U, A, I)$ as well as meet and join operators is denoted by $L_{P}(U, A, I)$ and is called the property oriented concept lattice of the formal context $(U, A, I)$.

Property 1 ([20]) Let $(U, A, I)$ be a formal context, $X, X_{1}, X_{2}$ be object sets, and $B, B_{1}, B_{2}$ be attribute sets, then the following results hold.

(1) If $X_{1} \subseteq X_{2}$, then $X_{1}^{\square} \subseteq X_{2}^{\square}$, and $X_{1}^{\diamond} \subseteq X_{2}^{\diamond}$; If $B_{1} \subseteq B_{2}$, then $B_{1}^{\square} \subseteq B_{2}^{\square}$, and $B_{1}^{\diamond} \subseteq B_{2}^{\diamond}$.

(2) $X^{\square \diamond} \subseteq X \subseteq X^{\diamond \square}$, and $B^{\natural \diamond} \subseteq B \subseteq B^{\diamond \sqsupset}$.

(3) $X^{\square}=X^{\square \vee \square}$, and $B=B^{\square \diamond \square} ; X^{\diamond}=X^{\diamond \square \diamond}$, and $B^{\diamond}=B^{\diamond \square \diamond}$.

(4) $\left(X_{1} \cap X_{2}\right)^{\square}=X_{1}^{\square} \cap X_{2}^{\square}$, and $\left(B_{1} \cap B_{2}\right)^{\square}=B_{1}^{\square} \cup B_{2}^{\square}$.

(5) $\left(X_{1} \cup X_{2}\right)^{\diamond}=X_{1}^{\diamond} \cup X_{2}^{\diamond}$, and $\left(B_{1} \cup B_{2}\right)^{\diamond}=B_{1}^{\diamond} \cup B_{2}^{\diamond}$.

An information system is a triple $(U, A, F)$, where $U$ is the finite set of objects and $A$ is the finite set of attributes, $F$ is a set of functions between $U$ and $A$. Obviously, a formal context is a special two-valued information system.

In [8], Novotny defined a congruence relation on the attribute power set $\mathrm{P}(A)$ and a dependence space in information systems.

Definition 4 ([8]) Let $(U, A, F)$ be an information system. $\mathrm{K}$ is an equivalence relation on $\mathrm{P}(A)$. Then, $\mathrm{K}$ is called a congruence relation on $(\mathrm{P}(A), \cup)$, whenever it satisfies the following condition :if $\left(B_{1}, C_{1}\right) \in \mathrm{K},\left(B_{2}, C_{2}\right) \in \mathrm{K}$, then $\left(B_{1} \cup B_{2}, C_{1} \cup C_{2}\right) \in \mathrm{K}$ for any $B_{1}, B_{2}, C_{1}, C_{2} \subseteq A$.

Definition 5 ([8]) Let $A$ be a finite nonempty set, and $\mathrm{K}$ a congruence relation ${ }_{\text {on }}(\mathrm{P}(A), \cup)$. Then the ordered pair $(A, \mathrm{~K})$ is said to be a dependence space.

Definition 6 ([2]) A closure operator is a mapping $C: 2^{U} \rightarrow 2^{U}$ such that $X \subseteq C(X)$; if $X \subseteq Y$, then $C(X) \subseteq C(Y)$; and $C(X)=C(C(X))$ for all $X, Y \subseteq U$.

\section{Notion and Approach to Attribute Reduction in Formal Contexts}

Let $(U, A, I)$ be a formal context. For any $B \subseteq A$, a binary relation on the object power set $\mathrm{P}(U)$ is defined as $R^{\diamond B}=\left\{(X, Y) \in \mathrm{P}(U) \times \mathrm{P}(U) \mid X^{\diamond B}=Y^{\diamond B}\right\}$. It is easy to prove that $R^{\diamond B}$ is a congruence relation on $(\mathrm{P}(U), \cup)$ and $\left(U, R^{\diamond B}\right)$ is a dependence space by Property 1 (5). We then define $[X]_{R^{\diamond B}}=\left\{Y \in \mathrm{P}(U) \mid(X, Y) \in R^{\diamond B}\right\}$, the congruence class determined by $X$ with respect to the congruence relation $R^{\diamond B}$, and $C_{R^{\circ B}}(X)=\bigcup\left\{Y \mid Y \in[X]_{R^{O B}}\right\}$ the maximum element of the congruence class $[X]_{R^{\circ B}}$.

By Property 1 and the definition of $C_{R^{\curvearrowright B}}(X)$, we have the following Lemma 1 immediately. 
Lemma 1 Let $(U, A, I)$ be a formal context. For any $X, Y, Z \in \mathrm{P}(U)$ and $B \subseteq A$, the following statements hold: $(1)\left(C_{R^{\diamond B}}(X), X\right) \in R^{\diamond B}$; (2) $C_{R^{\diamond B}}$ is a closure operator; (3) $C_{R^{\diamond B}}(X)=X^{\diamond B \square B}$.

Let $L_{P U}(U, A, I)=\left\{X \mid(X, B) \in L_{P}(U, A, I)\right\}$ be the set of all extents in the property oriented concept lattice $L_{P}(U, A, I)$. By Lemma 1 and Property 1 (3), we have $\left(C_{R^{\diamond B}}(X), X^{\diamond B}\right) \in L_{P}\left(U, B, I_{B}\right) \quad$ for $\quad$ any $\quad X \subseteq U$ and $B \subseteq A \quad$ that is $C_{R^{\diamond B}}(X) \in L_{P U}\left(U, B, I_{B}\right)$. On the other hand, if $X \in L_{P U}\left(U, B, I_{B}\right)$, then $X=X^{\diamond B \square B}$. According to Lemma 1 (3), we have $X=C_{R^{\circ B}}(X)$. Hence $L_{P U}\left(U, B, I_{B}\right)=\left\{C_{R^{\diamond B}}(X) \mid X \subseteq U\right\}$

Example 1 Table 1 gives a formal context $(U, A, I)$ with $U=\{1,2,3,4,5\}$ and $^{A}=\{a, b, c, d, e\}$. Figure 1 shows the property oriented concept lattice $L_{P}(U, A, I)$.

Table 1. A Formal Context $(U, A, I)$

\begin{tabular}{llllll}
\hline$U$ & $a$ & $b$ & $c$ & $d$ & $e$ \\
\hline 1 & 0 & 1 & 0 & 1 & 0 \\
2 & 1 & 0 & 1 & 0 & 1 \\
3 & 1 & 1 & 0 & 0 & 1 \\
4 & 0 & 1 & 1 & 1 & 0 \\
5 & 1 & 0 & 0 & 0 & 1 \\
\hline
\end{tabular}

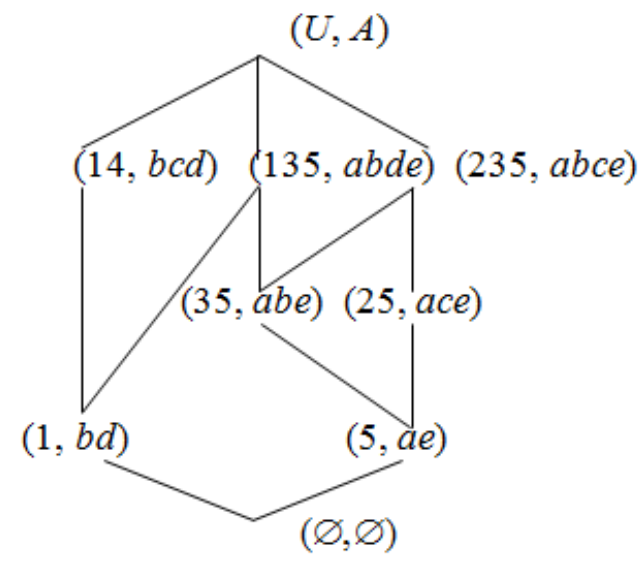

Figure 1. The Property Oriented Concept Lattice ${ }^{L_{P}}(U, A, I)$

According to the definition of the congruence class, all congruence classes of the context $(U, A, I)$ are calculated as

$[\{1\}]_{R^{\circ}}=\{\{1\}\},[\{2\}]_{R^{\circ}}=\{\{2\},\{2,5\}\},[\{3\}]_{R^{\circ}}=\{\{3\},\{3,5\}\},[\{4\}]_{R^{\circ}}=\{\{4\},\{1,4\}\}$,

$[\{5\}]_{R^{\circ}}=\{\{5\}\},[\{1,3\}]_{R^{\circ}}=\{\{1,3\},\{1,5\},\{1,3,5\}\},[\{2,3\}]_{R^{\circ}}=\{\{2,3\},\{2,3,5\}\},[\varnothing]_{R^{\circ}}=\{\varnothing\}$,

$[\{1,2\}]_{R^{0}}=\{\{1,2\},\{2,4\},\{3,4\},\{4,5\},\{1,2,3\},\{1,2,4\},\{1,2,5\},\{1,3,4\},\{1,4,5\},\{2,3,4\}$,

$$
\{2,4,5\},\{3,4,5\},\{1,2,3,4\},\{1,2,3,5\},\{1,2,4,5\},\{1,3,4,5\},\{2,3,4,5\}, U\} .
$$

Since $C_{R^{\circ B}}(X)=\bigcup\left\{Y \mid Y \in[X]_{R^{\circ B}}\right\}$, we obtain 


$$
\begin{aligned}
& C_{R^{\diamond}}(\{1\})=\{1\}, C_{R^{\diamond}}(\{2\})=\{2,5\}, C_{R^{\diamond}}(\{3\})=\{3,5\}, C_{R^{\vartheta}}(\{4\})=\{1,4\}, C_{R^{\diamond}}(\{5\})=\{5\}, \\
& C_{R^{0}}(\{1,3\})=\{1,3,5\}, C_{R^{0}}(\{2,3\})=\{2,3,5\}, C_{R^{0}}(\varnothing)=\varnothing, C_{R^{0}}(\{1,2\})=U \text {. } \\
& \left\{C_{R^{0}}(X) \mid X \subseteq U\right\}=\{\{1\},\{2,5\},\{3,5\},\{1,4\},\{5\},\{1,3,5\},\{2,3,5\}, \varnothing, U\}=L_{P U}(U, A, I) .
\end{aligned}
$$

Lemma 2 Let $(U, A, I)$ be a formal context. For any $X, Y, Z \in \mathrm{P}(U)$ and $B \subseteq A$, if $X \subseteq Y \subseteq Z$ and $(X, Z) \in R^{\diamond B}$, then $(X, Y) \in R^{\diamond B}$ and $(Y, Z) \in R^{\diamond B}$ hold.

Since $R^{\diamond B}$ is a congruence relation, Lemma 2 can be easily proved by the definition of congruence relation.

Lemma 3 Let $\left(U, A_{1}, I_{1}\right)$ and $\left(U, A_{2}, I_{2}\right)$ be two formal contexts with the same object set. For any $X \subseteq U$, if $L_{P U}\left(U, A_{2}, I_{2}\right) \subseteq L_{P U}\left(U, A_{1}, I_{1}\right)$, then

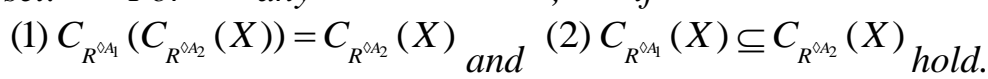

Proof. (1) Since $C_{R^{\circ A_{2}}}(X) \in L_{P U}\left(U, A_{2}, I_{2}\right)$ for any $X \subseteq U$, we obtain that if $L_{P U}\left(U, A_{2}, I_{2}\right) \subseteq L_{P U}\left(U, A_{1}, I_{1}\right)$, then $C_{R^{\Delta \lambda_{2}}}(X) \in L_{P U}\left(U, A_{1}, I_{1}\right)$ which implies that $\left(C_{R^{\triangleright A_{2}}}(X)\right)^{\nabla A_{1} \square A_{1}}=C_{R^{\curvearrowright A_{2}}}(X)$. Therefore, (i) is concluded considering that $C_{R^{\circ A_{1}}}\left(C_{R^{\circ A_{2}}}(X)\right)=\left(C_{R^{\circ A_{2}}}(X)\right)^{\nabla A_{\square} \square A_{1}}$ based on Lemma 1 (3)

(2) Since $C_{R^{\curvearrowright A_{1}}}$ is a closure operator, we have $C_{R^{\otimes A_{1}}}(X) \subseteq C_{R^{\circ A_{1}}}\left(C_{R^{\circ \alpha_{2}}}(X)\right)$. Thus, $C_{R^{\circ A_{1}}}(X) \subseteq C_{R^{\circ A_{2}}}(X)$ follows directly from (i). The proof is completed.

The following Theorem 1 shows us the relationship among property oriented concept lattices and the corresponding congruence relations.

Theorem 1 Let $\left(U, A_{1}, I_{1}\right)$ and $\left(U, A_{2}, I_{2}\right)$ be two formal contexts with the same object set. Then $L_{P U}\left(U, A_{2}, I_{2}\right) \subseteq L_{P U}\left(U, A_{1}, I_{1}\right)$ if and only if $R^{\diamond A_{1}} \subseteq R^{\diamond A_{2}}$.

Proof. (Sufficiency.) Assume that the condition $L_{P U}\left(U, A_{2}, I_{2}\right) \subseteq L_{P U}\left(U, A_{1}, I_{1}\right)$ does not hold, then there exists $X \in L_{P U}\left(U, A_{2}, I_{2}\right)$ such that $X \notin L_{P U}\left(U, A_{1}, I_{1}\right)$. Thus, $C_{R^{\diamond A_{2}}}(X)=X \subset C_{R^{\diamond A_{1}}}(X) \quad$ is concluded. Since $R^{\diamond A_{1}} \subseteq R^{\diamond A_{2}}$ is equivalent to $[X]_{R^{\triangleright A_{1}}} \subseteq[X]_{R^{\otimes A_{2}}}$ for any $X \subseteq U$, we have $C_{R^{\otimes A_{1}}}(X) \subseteq C_{R^{\otimes \alpha_{2}}}(X)$, which is a contradiction to $C_{R^{\circ A_{2}}}(X) \subset C_{R^{\circ A_{1}}}(X)$. Consequently, $L_{P U}\left(U, A_{2}, I_{2}\right) \subseteq L_{P U}\left(U, A_{1}, I_{1}\right)$ holds.

(Necessity.) Assume that $R^{\diamond A_{1}} \subseteq R^{\diamond A_{2}}$ does not hold, then there exits $X \subseteq U$ such that $[X]_{R^{\circ A_{1}}} \subseteq[X]_{R^{\circ \alpha_{2}}}$ does not hold. Thus, there exists $Y \in[X]_{R^{\circ A_{1}}}$ such that $Y \notin[X]_{R^{\otimes \alpha_{2}}}$. We prove the theorem from two cases $X \in L_{P U}\left(U, A_{1}, I_{1}\right)$ or $X \notin L_{P U}\left(U, A_{1}, I_{1}\right)$.

Firstly, we suppose $X \in L_{P U}\left(U, A_{1}, I_{1}\right)$. Since $Y \in[X]_{R^{\curvearrowright A_{1}}}$ and $Y \notin[X]_{R^{\curvearrowright \alpha_{2}}}$, we obtain $Y \subset C_{R^{\circ A_{1}}}(Y)=X$. Combining with $C_{R^{\diamond \alpha_{1}}}(Y) \subseteq C_{R^{\circ \alpha_{2}}}(Y)$ by Lemma 3 (2), we have $Y \subset X=C_{R^{\diamond A_{1}}}(Y) \subseteq C_{R^{\otimes A_{2}}}(Y)$. Due to Lemma 2, $(Y, X) \in R^{\diamond A_{2}}$, which is a contradiction to $Y \notin[X]_{R^{\diamond \alpha_{2}}}$. Therefore, $[X]_{R^{\diamond A_{1}}} \subseteq[X]_{R^{\diamond A_{2}}}$ holds. That is $R^{\diamond A_{1}} \subseteq R^{\diamond A_{2}}$.

Secondly, we suppose $X \notin L_{P U}\left(U, A_{1}, I_{1}\right)$. According to the above discussions, we have $\left[C_{R^{\triangleright A_{1}}}(X)\right]_{R^{\curvearrowright A_{1}}} \subseteq\left[C_{R^{\curvearrowright A_{1}}}(X)\right]_{R^{\curvearrowright \alpha_{2}}} \quad$ due $\quad$ to $\quad C_{R^{\triangleright A_{1}}}(X) \in L_{P U}\left(U, A_{1}, I_{1}\right)$ Since $Y \in[X]_{R^{\curvearrowright A_{1}}}=\left[C_{R^{\diamond \alpha_{1}}}(X)\right]_{R^{\diamond A_{1}}}$, it is evident that $Y \subseteq C_{R^{\diamond A_{1}}}(X)$ and $Y \in\left[C_{R^{\otimes A_{1}}}(X)\right]_{R^{\curvearrowright A_{2}}}$. 
Combining with $C_{R^{\otimes A_{1}}}(X) \subseteq C_{R^{\otimes \alpha_{2}}}(X)$, we have $Y \subseteq C_{R^{\otimes A_{2}}}(X)$. Since $C_{R^{\diamond \alpha_{2}}}$ is a closure operator, we have $C_{R^{\otimes A_{2}}}(X) \subseteq C_{R^{\otimes \alpha_{2}}}\left(C_{R^{\otimes A_{1}}}(X)\right)$. And $Y \in\left[C_{R^{\otimes A_{1}}}(X)\right]_{R^{\otimes A_{2}}}$ implies $C_{R^{0 A_{2}}}(Y)=C_{R^{0 A_{2}}}\left(C_{R^{\circ A_{1}}}(X)\right)$. Thus, $\quad Y \subseteq C_{R^{\circ A_{2}}}(X) \subseteq C_{R^{\alpha_{2}}}(Y) . \quad$ By Lemma 2, $\left(Y, C_{R^{\diamond A_{2}}}(X)\right) \in R^{\diamond A_{2}}$ holds. That is, $(Y, X) \in R^{\diamond A_{2}}$, which is a contradiction to $Y \notin[X]_{R^{\diamond A_{2}}}$. Therefore, $[X]_{R^{\triangleright A_{1}}} \subseteq[X]_{R^{\diamond \alpha_{2}}}$ is concluded. That is $R^{\diamond A_{1}} \subseteq R^{\diamond A_{2}}$. The proof is completed.

Corollary 1 Let $\left(U, A_{1}, I_{1}\right)$ and $\left(U, A_{2}, I_{2}\right)$ be two formal contexts with the same object set. Then $L_{P U}\left(U, A_{2}, I_{2}\right)=L_{P U}\left(U, A_{1}, I_{1}\right)$ if and only if $R^{\diamond A_{1}}=R^{\diamond A_{2}}$.

In the following section, we develop the notion of attribute reduction in a property oriented concept lattice of a formal context based on the congruence relations and then define an approach to attribute reduction.

Definition 7 Let $(U, A, I)$ be a formal context. For any $B \subseteq A$, if $R^{\diamond A}=R^{\diamond B}$, then $B$ is called a consistent set of the property oriented concept lattice $L_{P}(U, A, I)$. Further, for any $b \in B$ if $R^{\diamond A} \neq R^{\diamond B-\{b\}}$, then $B$ is called an attribute reduct of $L_{P}(U, A, I)$.

Definition 7 shows that consistent sets preserve all original congruence classes determined by the original attribute set. By Corollary 1 and Definition 7, we can obtain the following result directly.

Lemma 4 Let $(U, A, I)$ be a formal context. For any $B \subseteq A$ and $b \in B$, we have the following two statements: (1) $B$ is a consistent set if and only if $L_{P U}(U, A, I)=L_{P U}\left(U, B, I_{B}\right)$.

(2) $B$ is an attribute reduct if and only if $L_{P U}(U, A, I)=L_{P U}\left(U, B, I_{B}\right)$, and $L_{P U}(U, A, I) \neq L_{P U}\left(U, B-\{b\}, I_{B-\{b\}}\right)$

For convenience, we use $R^{\diamond a}$ instead of $R^{\diamond\{a\}}$ for any $a \in A$.

Definition 8 Let $(U, A, I)$ be a formal context. For any $X_{i}, X_{j} \subseteq U$, we define

$$
D\left(\left[X_{i}\right]_{R^{\diamond A}},\left[X_{j}\right]_{R^{\diamond A}}\right)=\left\{a \in A \mid\left(X_{i}, X_{j}\right) \notin R^{\diamond a}\right\}
$$

Then $D\left(\left[X_{i}\right]_{R^{\curvearrowright A}},\left[X_{j}\right]_{R^{\curvearrowright \Lambda}}\right)$ is called the discernibility attribute set between $\left[X_{i}\right]_{R^{\circ A}}$ and $\left[X_{j}\right]_{R^{\curvearrowright A}}$, and $\Delta=\left(D\left(\left[X_{i}\right]_{R^{\circ A}},\left[X_{j}\right]_{R^{\diamond A}}\right) \mid X_{i}, X_{j} \in \mathrm{P}(U)\right)$ is called the discernibility matrix.

Theorem 2 Let $(U, A, I)$ be a formal context. For any $X_{i}, X_{j} \subseteq U$, we have

$$
D\left(\left[X_{i}\right]_{R^{\curvearrowright A}},\left[X_{j}\right]_{R^{\curvearrowright A}}\right)=B_{i} \cup B_{j}-B_{i} \cap B_{j},
$$

where $\left(C_{R^{\otimes A}}\left(X_{i}\right), B_{i}\right) \in L_{P}(U, A, I)$ and $\left(C_{R^{\circ A}}\left(X_{j}\right), B_{j}\right) \in L_{P}(U, A, I)$.

Proof. Since $\left(C_{R^{\wedge A}}\left(X_{i}\right), B_{i}\right) \in L_{P}(U, A, I)$ and $\left(C_{R^{\wedge A}}\left(X_{j}\right), B_{j}\right) \in L_{P}(U, A, I)$ for any $X_{i}, X_{j} \subseteq U$, we obtain $a \in D\left(\left[X_{i}\right]_{R^{\diamond A}},\left[X_{j}\right]_{R^{\diamond A}}\right) \Leftrightarrow\left(X_{i}, X_{j}\right) \notin R^{\diamond a} \Leftrightarrow X_{i}^{\diamond a} \neq X_{j}^{\diamond a}$

$\Leftrightarrow\left(C_{R^{\diamond A}}\left(X_{i}\right)\right)^{\diamond a} \neq\left(C_{R^{\otimes A}}\left(X_{j}\right)\right)^{\diamond a} \Leftrightarrow a \in B_{i} \cup B_{j}-B_{i} \cap B_{j}$,

The proof is completed.

Property 2 Let $(U, A, I)$ be a formal context. For any $X_{i}, X_{j} \subseteq U$ and $B \subseteq A$, the following properties hold:

(1) $D\left(\left[X_{i}\right]_{R^{\circ A}},\left[X_{i}\right]_{R^{\curvearrowright A}}\right)=\varnothing$. 
(2) $D\left(\left[X_{i}\right]_{R^{\diamond A}},\left[X_{j}\right]_{R^{\diamond A}}\right)=D\left(\left[X_{j}\right]_{R^{\curvearrowright A}},\left[X_{i}\right]_{R^{\diamond A}}\right)$.

(3) $D\left(\left[X_{i}\right]_{R^{\curvearrowright A}},\left[X_{j}\right]_{R^{\curvearrowright A}}\right) \subseteq D\left(\left[X_{i}\right]_{R^{\circ A}},\left[X_{k}\right]_{R^{\circ A}}\right) \cup D\left(\left[X_{k}\right]_{R^{\curvearrowright A}},\left[X_{j}\right]_{R^{\circ A}}\right)$.

(4) $D\left(\left[X_{i}\right]_{R^{\curvearrowright B}},\left[X_{j}\right]_{R^{\curvearrowright B}}\right)=D\left(\left[X_{i}\right]_{R^{\curvearrowright A}},\left[X_{j}\right]_{R^{\curvearrowright A}}\right) \cap B$.

Theorem 3 Let $(U, A, I)$ be a formal context. For any nonempty attribute $\operatorname{set} B$, we have that $B$ is a consistent set if and only if $B \cap D\left(\left[X_{i}\right]_{R^{\circ A}},\left[X_{j}\right]_{R^{\circ A}}\right) \neq \varnothing$ holds for any $D\left(\left[X_{i}\right]_{R^{\curvearrowright A}},\left[X_{j}\right]_{R^{\diamond A}}\right) \neq \varnothing$.

Proof. (Sufficiency.) Since $B \cap D\left(\left[X_{i}\right]_{R^{\circ A}},\left[X_{j}\right]_{R^{\circ A}}\right) \neq \varnothing$ holds for any $D\left(\left[X_{i}\right]_{R^{\circ A}},\left[X_{j}\right]_{R^{\circ A}}\right) \neq \varnothing$, we have that $D\left(\left[X_{i}\right]_{R^{\circ B}},\left[X_{j}\right]_{R^{O B}}\right) \neq \varnothing$ for any $D\left(\left[X_{i}\right]_{R^{\circ A}},\left[X_{j}\right]_{R^{\circ A}}\right) \neq \varnothing$. On the other hand, we have that if $D\left(\left[X_{i}\right]_{R^{\circ B}},\left[X_{j}\right]_{R^{\diamond B}}\right) \neq \varnothing$, then $D\left(\left[X_{i}\right]_{R^{\circ A}},\left[X_{j}\right]_{R^{\curvearrowright A}}\right) \neq \varnothing \quad$ by Property 2 (4). Thus, $D\left(\left[X_{i}\right]_{R^{\circ B}},\left[X_{j}\right]_{R^{\circ B}}\right) \neq \varnothing$ if and only if $D\left(\left[X_{i}\right]_{R^{\circ A}},\left[X_{j}\right]_{R^{\circ A}}\right) \neq \varnothing$, which implies that $\left[X_{i}\right]_{R^{\curvearrowright A}} \cap\left[X_{j}\right]_{R^{\curvearrowright \Lambda}}=\varnothing$ if and only if $\left[X_{i}\right]_{R^{\diamond B}} \cap\left[X_{j}\right]_{R^{\diamond B}}=\varnothing$ for any $X_{i}, X_{j} \subseteq U$. Therefore, $R^{\diamond A}=R^{\diamond B}$, i.e. $B$ is a consistent set.

(Necessity.) If $B$ is a consistent set, then $R^{\diamond A}=R^{\diamond B}$ which implies $[X]_{R^{\diamond A}}=[X]_{R^{\diamond B}}$ for any $X \subseteq U$. Thus we have $\left[X_{i}\right]_{R^{\curvearrowright A}} \cap\left[X_{j}\right]_{R^{\curvearrowright A}}=\varnothing$ if and only if $\left[X_{i}\right]_{R^{\circ B}} \cap\left[X_{j}\right]_{R^{\circ B}}=\varnothing$. Hence $D\left(\left[X_{i}\right]_{R^{\curvearrowright A}},\left[X_{j}\right]_{R^{\curvearrowright A}}\right) \neq \varnothing$ if and only if $D\left(\left[X_{i}\right]_{R^{\circ B}},\left[X_{j}\right]_{R^{\circ B}}\right) \neq \varnothing$. Therefore, for any $D\left(\left[X_{i}\right]_{R^{\circ A}},\left[X_{j}\right]_{R^{\circ A}}\right) \neq \varnothing$, we have $B \cap D\left(\left[X_{i}\right]_{R^{\circ A}},\left[X_{j}\right]_{R^{\circ A}}\right) \neq \varnothing$ due to $D\left(\left[X_{i}\right]_{R^{\circ B}},\left[X_{j}\right]_{R^{\circ B}}\right)=D\left(\left[X_{i}\right]_{R^{\circ A}},\left[X_{j}\right]_{R^{\circ A}}\right) \cap B$.

Example 2 Table 2 shows the discernibility matrix $\Delta_{o f}(U, A, I)$ in Table 1.

Table 2. The Discernibility Matrix $\Delta$ of $(U, A, I)$

\begin{tabular}{cccccccccc}
\hline & $U$ & $\{1,4\}$ & $\{1,3,5\}$ & $\{2,3,5\}$ & $\{3,5\}$ & $\{2,5\}$ & $\{1\}$ & $\{5\}$ & $\varnothing$ \\
\hline$U$ & $\varnothing$ & & & & & & & & \\
$\{1,4\}$ & $\{a, e\}$ & $\varnothing$ & & & & & & & \\
$\{1,3,5\}$ & $\{c\}$ & $\{a, c, e\}$ & $\varnothing$ & & & & & & \\
$\{2,3,5\}$ & $\{d\}$ & $\{a, d, e\}$ & $\{c, d\}$ & $\varnothing$ & & & & & \\
$\{3,5\}$ & $\{c, d\}$ & $\{a, c, d, e\}$ & $\{d\}$ & $\{c\}$ & $\varnothing$ & & & & \\
$\{2,5\}$ & $\{b, d\}$ & $\{a, b, d, e\}$ & $\{b, c, d\}$ & $\{b\}$ & $\{b, c\}$ & $\varnothing$ & & & \\
$\{1\}$ & $\{a, c, e\}$ & $\{c\}$ & $\{a, e\}$ & $\{a, c, d, e\}$ & $\{a, d, e\}$ & $A$ & $\varnothing$ & & \\
$\{5\}$ & $\{b, c, d\}$ & $A$ & $\{b, d\}$ & $\{b, c\}$ & $\{b\}$ & $\{c\}$ & $\{a, b, d, e\}$ & $\varnothing$ & \\
$\varnothing$ & $A$ & $\{b, c, d\}$ & $\{a, b, d, e\}$ & $\{a, b, c, e\}$ & $\{a, b, e\}$ & $\{a, c, e\}$ & $\{b, d\}$ & $\{a, e\}$ & $\varnothing$ \\
\hline
\end{tabular}



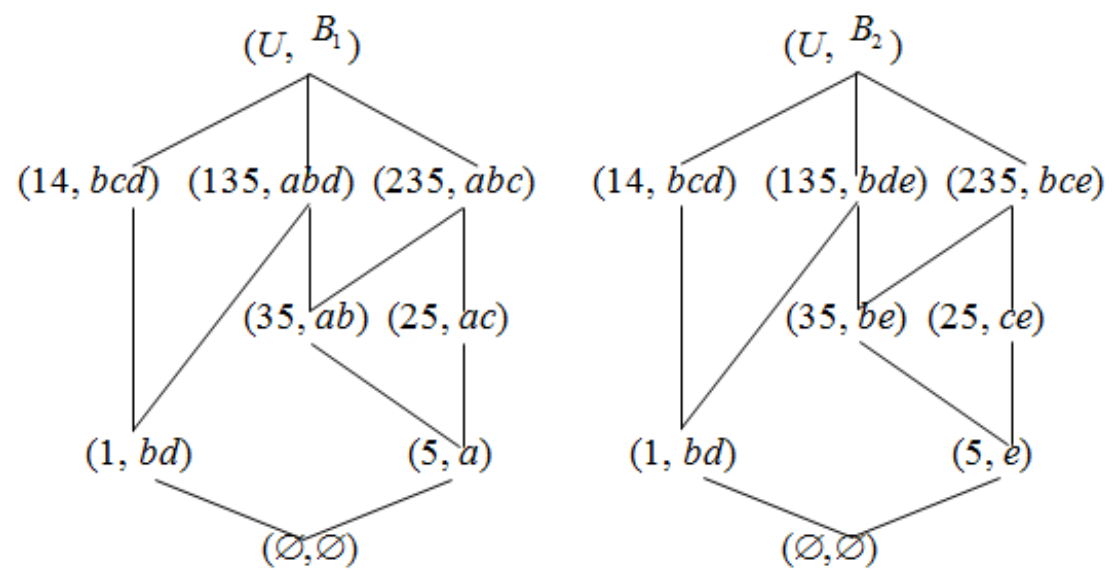

Figure 2. The Property Oriented Concept Lattices ${ }^{L_{P}}\left(U, B_{1}, I_{B_{1}}\right)$ and

$$
L_{P}\left(U, B_{2}, I_{B_{2}}\right)
$$

According to Theorem 3 and Table 2, $B_{1}=\{a, b, c, d\}$ and $B_{2}=\{b, c, d, e\}$ are two attribute reducts of $(U, A, I)$. Figure 2 shows the property oriented concept lattices $L_{P}\left(U, B_{1}, I_{B_{1}}\right)$ and $L_{P}\left(U, B_{2}, I_{B_{2}}\right)$ respectively. It is easy to see that the three property oriented concept lattices $L_{P}(U, A, I), L_{P}\left(U, B_{1}, I_{B_{1}}\right)$ and $L_{P}\left(U, B_{2}, I_{B_{2}}\right)$ are isomorphic to each other.

\section{Notion and Approach to Attribute Reduction in Formal Decision Contexts}

In this section, we develop the notion of attribute reduction in formal decision contexts based on the congruence relations and then define an approach to attribute reduction.

Definition 9 Let $(U, A, I)$ and $(U, C, J)$ be two formal contexts with the same object set. $(U, A, I, C, J)$ is called a formal decision context, where $I \subseteq U \times A, J \subseteq U \times C$ and $A \cap C=\varnothing . A$ and $C$ are called condition attribute set and decision attribute set respectively.

Definition 10 Let $(U, A, I, C, J)$ be a formal decision context. $(U, A, I, C, J)$ is said to be consistent if $R^{\diamond A} \subseteq R^{\diamond C}$. Otherwise, it is said to be inconsistent. Where $R^{\diamond C}=\left\{(X, Y) \in P(U) \times P(U) \mid X^{\diamond C}=Y^{\diamond C}\right\}$.

Definition 11 Let $(U, A, C, J)$ be a formal decision context. For any $B \subseteq A$, if $R^{\diamond B} \subseteq R^{\diamond C}$, then $B$ is called a consistent set of the formal decision context $(U, A, C, J)$. Further, if $R^{\diamond B-\{b\}} \subseteq R^{\diamond C}$ does not hold for any $b \in B$, then $B$ is called an attribute reduct of $^{(U, A, C, J)}$.

By Theorem 1 and Definition 11, we can obtain the following result directly.

Lemma 5 Let $(U, A, I, C, J)$ be a formal context. For any $B \subseteq A$, we have the following two statements: (1) $B$ is a consistent set if and only if $L_{P U}(U, C, J) \subseteq L_{P U}\left(U, B, I_{B}\right)$. 
(2) $B$ is an attribute reduct if and only if $L_{P U}(U, C, J) \subseteq L_{P U}\left(U, B, I_{B}\right)$, and $L_{P U}(U, C, J) \subseteq L_{P U}\left(U, B-\{b\}, I_{B-\{b\}}\right)$ does not hold for any $b \in B$.

Example 3 Table 3 gives a formal decision context $(U, A, I, C, J)$ with $U=\{1,2,3,4,5\} \quad A=\{a, b, c, d, e\}$ and $C=\{g, h, i\}$. Here the formal context $(U, A, I)$ is the same with that in Example 1. And Figure 3 shows the property oriented concept lattice $L_{P}(U, C, J)$.

Table 3. A Formal Decision Context $(U, A, I, C, J)$

\begin{tabular}{llllll|lll}
\hline$U$ & $a$ & $b$ & $c$ & $d$ & $e$ & $g$ & $h$ & $i$ \\
\hline 1 & 0 & 1 & 0 & 1 & 0 & 0 & 1 & 0 \\
2 & 1 & 0 & 1 & 0 & 1 & 1 & 0 & 1 \\
3 & 1 & 1 & 0 & 0 & 1 & 1 & 1 & 0 \\
4 & 0 & 1 & 1 & 1 & 0 & 0 & 1 & 1 \\
5 & 1 & 0 & 0 & 0 & 1 & 1 & 0 & 0 \\
\hline
\end{tabular}

In Example 1, we have obtained all congruence classes of the context $(U, A, I)$ : $[\{1\}]_{R^{\circ}}=\{\{1\}\},[\{2\}]_{R^{\circ}}=\{\{2\},\{2,5\}\},[\{3\}]_{R^{0}}=\{\{3\},\{3,5\}\},[\{4\}]_{R^{\circ}}=\{\{4\},\{1,4\}\}$, $[\{5\}]_{R^{\circ}}=\{\{5\}\},[\{1,3\}]_{R^{\circ}}=\{\{1,3\},\{1,5\},\{1,3,5\}\},[\{2,3\}]_{R^{\circ}}=\{\{2,3\},\{2,3,5\}\},[\varnothing]_{R^{\circ}}=\{\varnothing\}$, $[\{1,2\}]_{R^{\circ}}=\{\{1,2\},\{2,4\},\{3,4\},\{4,5\},\{1,2,3\},\{1,2,4\},\{1,2,5\},\{1,3,4\},\{1,4,5\},\{2,3,4\}$,

$$
\{2,4,5\},\{3,4,5\},\{1,2,3,4\},\{1,2,3,5\},\{1,2,4,5\},\{1,3,4,5\},\{2,3,4,5\}, U\} \text {. }
$$

And all congruence classes of $(U, C, J)$ are computed as:

$$
[\{1\}]_{R^{\circ C}}=\{\{1\}\},[\{2\}]_{R^{\circ c}}=\{\{2\},\{2,5\}\},[\{3\}]_{R^{\circ}}=\{\{3\},\{3,5\},\{1,3\},\{1,5\},\{1,3,5\}\},
$$

$$
\begin{gathered}
{[\{4\}]_{R^{\circ}}=\{\{4\},\{1,4\}\},[\{5\}]_{R^{\circ C}}=\{\{5\}\},[\varnothing]_{R^{\circ C}}=\{\varnothing\},} \\
{[\{1,2\}]_{R^{\circ C}}=\{\{1,2\},\{2,3\},\{2,4\},\{3,4\},\{4,5\},\{1,2,3\},\{1,2,4\},\{1,2,5\},\{1,3,4\},\{1,4,5\},} \\
\\
\{2,3,4\},\{2,3,5\},\{2,4,5\},\{3,4,5\},\{1,2,3,4\},\{1,2,3,5\},\{1,2,4,5\}, \\
\\
\{1,3,4,5\},\{2,3,4,5\}, U\} .
\end{gathered}
$$

Obviously, $C_{R^{\circ A}}(X) \subseteq C_{R^{\diamond C}}(X)$ for all $X \subseteq U$. Thus, the formal decision context $(U, A, I, C, J)$ is consistent. 


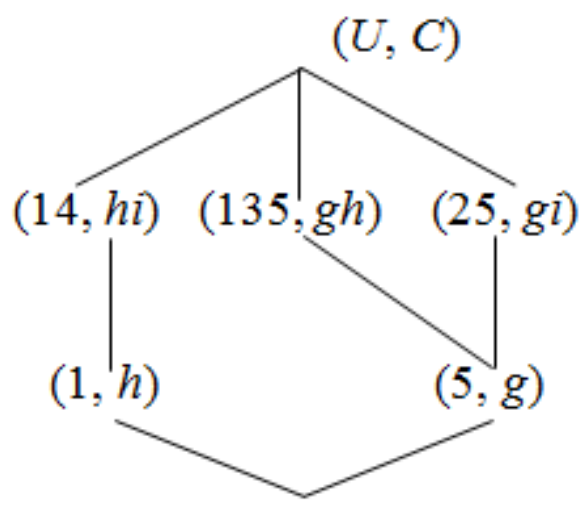

$(\varnothing, \varnothing)$

Figure 3. The Property Oriented Concept Lattice $L_{P}(U, C, J)$

Definition 12 Let $(U, A, I, C, J)$ be a consistent formal decision context. For any $X_{i}, X_{j} \subseteq U$, we define

$$
D_{d}\left(\left[X_{i}\right]_{R^{\curvearrowright A}},\left[X_{j}\right]_{R^{\diamond A}}\right)=\left\{\begin{array}{cc}
\left\{a \in A \mid\left(X_{i}, X_{j}\right) \notin R^{\diamond a}\right\},\left[X_{i}\right]_{R^{\circ C}} \cap\left[X_{j}\right]_{R^{\circ C}}=\varnothing \\
\varnothing, & {\left[X_{i}\right]_{R^{\circ C}} \cap\left[X_{j}\right]_{R^{\circ C}} \neq \varnothing}
\end{array}\right.
$$

Then $D_{d}\left(\left[X_{i}\right]_{R^{\circ A}},\left[X_{j}\right]_{R^{\circ A}}\right)$ is called the discernibility attribute set between $\left[X_{i}\right]_{R^{\circ A}}$

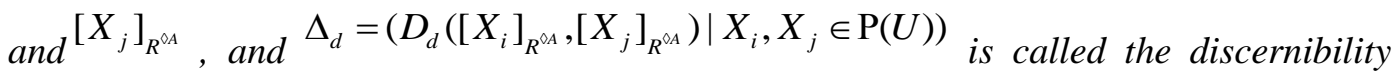
matrix of the formal decision context $(U, A, I, C, J)$.

Theorem 4 Let $(U, A, I, C, J)$ be a consistent formal decision context. For any $X_{i}, X_{j} \subseteq U$, we have $D_{d}\left(\left[X_{i}\right]_{R^{\circ A}},\left[X_{j}\right]_{R^{\circ A}}\right)=B_{i} \cup B_{j}-B_{i} \cap B_{j}$, if $\left[X_{i}\right]_{R^{\circ C}} \cap\left[X_{j}\right]_{R^{\circ C}}=\varnothing \quad$. Otherwise $\quad D_{d}\left(\left[X_{i}\right]_{R^{\circ A}},\left[X_{j}\right]_{R^{\circ A}}\right)=\varnothing$. where $\left.C_{R^{\circ A}}\left(X_{i}\right), B_{i}\right) \in L_{P}(U, A, I)$ and $\left(C_{R^{\circ A}}\left(X_{j}\right), B_{j}\right) \in L_{P}(U, A, I)$.

Property 3 Let $(U, A, I, C, J)$ be a consistent formal decision context. For any $X_{i}, X_{j} \subseteq U$ and $B \subseteq A$, the following properties hold:

(1) $D_{d}\left(\left[X_{i}\right]_{R^{\circ A}},\left[X_{i}\right]_{R^{\diamond A}}\right)=\varnothing$.

(2) $D_{d}\left(\left[X_{i}\right]_{R^{\curvearrowright A}},\left[X_{j}\right]_{R^{\circ A}}\right)=D_{d}\left(\left[X_{j}\right]_{R^{\curvearrowright A}},\left[X_{i}\right]_{R^{\circ A}}\right)$.

(3) $D_{d}\left(\left[X_{i}\right]_{R^{\circ B}},\left[X_{j}\right]_{R^{\circ B}}\right)=D_{d}\left(\left[X_{i}\right]_{R^{\circ A}},\left[X_{j}\right]_{R^{\circ A}}\right) \cap B$

Note that $D_{d}\left(\left[X_{i}\right]_{R^{\curvearrowright A}},\left[X_{j}\right]_{R^{\curvearrowright A}}\right) \subseteq D_{d}\left(\left[X_{i}\right]_{R^{\curvearrowright A}},\left[X_{k}\right]_{R^{\curvearrowright A}}\right) \cup D_{d}\left(\left[X_{k}\right]_{R^{\diamond A}},\left[X_{j}\right]_{R^{\curvearrowright A}}\right)$ may be not hold for any $X_{i}, X_{j}, X_{k} \subseteq U$. For example, if we select $X_{1}=\{3,5\}, X_{2}=\{1,3,5\}$ and $X_{3}=\{1,4\}$ in Table 3, then $D_{d}\left(\left[X_{1}\right]_{R^{\circ A}},\left[X_{2}\right]_{R^{\circ A}}\right)=\varnothing, \quad D_{d}\left(\left[X_{1}\right]_{R^{\circ A}},\left[X_{3}\right]_{R^{\circ A}}\right)=\{a, c, d, e\}$, $D_{d}\left(\left[X_{2}\right]_{R^{\circ A}},\left[X_{3}\right]_{R^{\circ A}}\right)=\{a, c, e\}$ according to Definition 12. Therefore,

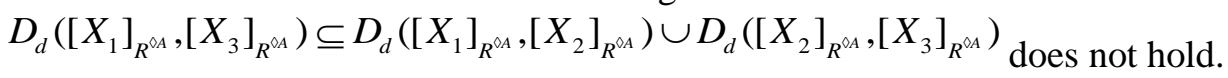


Theorem 5 Let $(U, A, I, C, J)$ be a formal context. For any nonempty attribute set $B$, we have that $B$ is a consistent set if and only if $B \cap D_{d}\left(\left[X_{i}\right]_{R^{\circ A}},\left[X_{j}\right]_{R^{\text {sA }}}\right) \neq \varnothing$ holds for any $D_{d}\left(\left[X_{i}\right]_{R^{\curvearrowright A}},\left[X_{j}\right]_{R^{\circ A}}\right) \neq \varnothing$.

The proofs of Theorems 4 and 5 are similar with those of Theorems 2 and 3 respectively.

Example 4 Table 4 gives the discernibility matrix of the formal decision context $(U, A, I, C, J)$ in Example 3.

Table 4. The Discernibility Matrix ${ }^{\Delta_{d}}$ of $(U, A, I, C, J)$

\begin{tabular}{cccccccccc}
\hline & $U$ & $\{1,4\}$ & $\{1,3,5\}$ & $\{2,3,5\}$ & $\{3,5\}$ & $\{2,5\}$ & $\{1\}$ & $\{5\}$ & $\varnothing$ \\
\hline$U$ & $\varnothing$ & & & & & & & & \\
$\{1,4\}$ & $\{a, e\}$ & $\varnothing$ & & & & & & & \\
$\{1,3,5\}$ & $\{c\}$ & $\{a, c, e\}$ & $\varnothing$ & & & & & & \\
$\{2,3,5\}$ & $\varnothing$ & $\{a, d, e\}$ & $\{c, d\}$ & $\varnothing$ & & & & & \\
$\{3,5\}$ & $\{c, d\}$ & $\{a, c, d, e\}$ & $\varnothing$ & $\{c\}$ & $\varnothing$ & & & & \\
$\{2,5\}$ & $\{b, d\}$ & $\{a, b, d, e\}$ & $\{b, c, d\}$ & $\{b\}$ & $\{b, c\}$ & $\varnothing$ & & & \\
$\{1\}$ & $\{a, c, e\}$ & $\{c\}$ & $\{a, e\}$ & $\{a, c, d, e\}$ & $\{a, d, e\}$ & $A$ & $\varnothing$ & & \\
$\{5\}$ & $\{b, c, d\}$ & $A$ & $\{b, d\}$ & $\{b, c\}$ & $\{b\}$ & $\{c\}$ & $\{a, b, d, e\}$ & $\varnothing$ & \\
$\varnothing$ & $A$ & $\{b, c, d\}$ & $\{a, b, d, e\}$ & $\{a, b, c, e\}$ & $\{a, b, e\}$ & $\{a, c, e\}$ & $\{b, d\}$ & $\{a, e\}$ & $\varnothing$ \\
\hline
\end{tabular}

According to Theorem 5 and Table $4, B_{3}=\{a, b, c\}$ and $B_{2}=\{b, c, e\}$ are attribute reducts of $(U, A, I, C, J)$. Figure 4 shows the property oriented concept lattices $L_{P}\left(U, B_{3}, I_{B_{3}}\right)$ and $L_{P}\left(U, B_{4}, I_{B_{4}}\right)$ respectively. Obviously, $L_{P U}\left(U, B_{3}, I_{B_{3}}\right)=L_{P U}\left(U, B_{4}, I_{B_{4}}\right)=L_{P U}(U, C, J)$
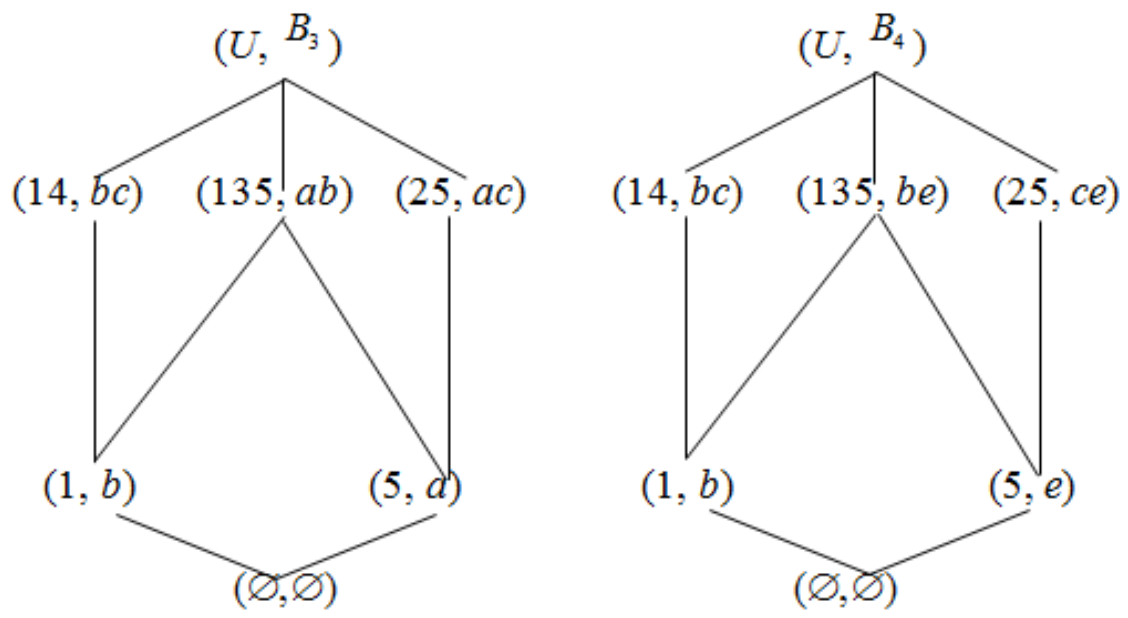

Figure 4. The Property Oriented Concept Lattices ${ }^{L_{P}\left(U, B_{3}, I_{B_{3}}\right)}$ and $L_{P}\left(U, B_{4}, I_{B_{4}}\right)$ 


\section{Conclusion}

This paper has developed notions and approaches to attribute reduction in property oriented concept lattices of a formal context and formal decision context based on congruence relations. Discernibility matrices have been subsequently defined to calculate all attribute reducts. Basing on the reduction method proposed in this paper, we can study knowledge reduction in property oriented concept lattices of inconsistent formal decision contexts in further research.

\section{Acknowledgements}

This work was supported by the Natural Science Foundation of China (Nos. 61202206 and 11202187).

\section{References}

[1] B. Ganter and R. Wille, "Formal Concept Analysis: Mathematical Foundations", Springer-Verlag, New York, (1999).

[2] G. Gediga and I. Duntsch, "Modal-style operators in qualitative data analysis", Proceedings of the 2002 IEEE International Conference on Data Mining, Maebashi, Japan, (2002).

[3] J. H. Li, C. L. Mei and Y. J. Lv, "Knowledge reduction in formal decision contexts based on an orderpreserving mapping", International Journal of General Systems, vol. 2, no. 41, (2012), pp. 143-161.

[4] J. H. Li, C. L. Mei and Y. J. Lv, "Incomplete decision contexts: approximate concept construction, rule acquisition and knowledge reduction", International Journal of Approximation Reasoning, vol. 1, no. 54, (2013), pp. 149-165.

[5] H. W. Liu, L. Liu and H. J. Zhang, "A fast pruning redundant rule method using Galois connection", Applied Soft Computing, vol. 11, (2011), pp. 130-137.

[6] M. Liu, M. W. Shao, W. X. Zhang and C. Wu, "Reduction method for concept lattices based on rough set theory and its application", Computers and Mathematics with Applications, vol. 53, (2007), pp. 1390-1410.

[7] J. S. Mi, Y. Leung and W. Z. Wu, "Approaches to attribute reduction in concept lattices induced by axialities”, Knowledge-Based Systems, vol. 23, (2010), pp. 504-511.

[8] M. Novotny, "Dependence spaces of information system", In Incomplete Information: Rough Set Analysis, Edited E. Orlowska, Physica-Verlag, Heidelberg-New York, (1998), pp. 193-246.

[9] X. Wang and J. M. Ma, "A novel approach to attribute reduction in concept lattices", Proceedings of the 1st International Conference on Rough Sets and Knowledge Technology, Chongqing, China, (2006).

[10] X. Wang and W. X. Zhang, "Relations of attribute reduction between object and property oriented concept lattices", Knowledge-Based Systems, vol. 5, no. 21, (2008), pp. 398-403.

[11] X. Wang, "Approaches to attribute reduction in concept lattices based on rough set theory", International Journal of Hybrid Information Technology, vol. 2, no. 5, (2012), pp. 67-79.

[12] X. Wang and W. Z. Wu, "Approximate reduction in inconsistent formal decision contexts", Proceedings of 2012 IEEE International Conference on Granular Computing, Hangzhou, China, (2012).

[13] X. Wang, "Construction of a unified model for formal contexts and formal decision contexts", International Journal of Database Theory and Application, vol. 2, no. 7, (2014), pp. 81-90.

[14] X. Wang and W. Z. Wu, "Attribute reduction in object oriented concept lattices based on congruence relations", Proceedings of the 9th International Conference on Rough Sets and Knowledge Technology, Shanghai, China, (2014).

[15] L. Wei, J. J. Qi and W. X. Zhang, "Attribute reduction theory of concept lattice based on decision formal contexts", Science in China Series F-Information Science vol. 7, no. 51, (2008), pp. 910-923.

[16] W. Z. Wu, Y. Leung and J. S. M, "Granular computing and knowledge reduction in formal contexts", IEEE Transactions Knowledge and Data Engineering, vol. 10, no. 21, (2009), pp. 1461-1474.

[17] W. X. Zhang, L. Wei and J. J. Qi, "Attribute reduction theory and approach to concept lattice", Science in China Series F-Information Science, vol. 6, no. 48, (2005), pp. 713-726.

[18] W. X. Zhang and G. F Qiu, "Uncertain Decision Making Based on Rough Sets", Tsinghua University Publishing House, Beijing, (2005).

[19] Y. Y. Yao, "Concept lattices in rough set theory", Proceedings of 2004 Annual Meeting of the North American Fuzzy Information Processing Society, Washington, D.C., USA, (2004).

[20] Y. Y. Yao, "A comparative study of formal concept analysis and rough set theory in data analysis", Proceedings of the 4th International Conference on Rough Sets and Current Trends in Computing, Uppsala, Sweden, (2004). 